\title{
BMJ Open Study protocol: characterising the clinical, epidemiological and aetiological aspects of leptospirosis in Sri Lanka: a hospital based clinico- epidemiological study
}

\author{
Suneth Agampodi, ${ }^{1}$ Janith Warnasekara, ${ }^{1}$ Dinesha Jayasundara, ${ }^{2}$ \\ Indika Senawirathna, ${ }^{3}$ Chandika Gamage, ${ }^{4}$ Senanayake Kularatne, ${ }^{5}$ \\ Sisira Siribaddana, ${ }^{6}$ Michael Maththias, ${ }^{7}$ Joseph Vinetz ${ }^{7}$
}

To cite: Agampodi S, Warnasekara J, Jayasundara D, et al. Study protocol: characterising the clinical, epidemiological and aetiological aspects of leptospirosis in Sri Lanka: a hospital based clinico-epidemiological study. BMJ Open 2019;9:e027850. doi:10.1136/ bmjopen-2018-027850

- Prepublication history for this paper is available online To view these files please visit the journal online (http://dx.doi org/10.1136/bmjopen-2017016527).

Received 20 November 2018 Revised 11 July 2019 Accepted 19 August 2019
Check for updates

(C) Author(s) (or their employer(s)) 2019. Re-use permitted under CC BY-NC. No commercial re-use. See rights and permissions. Published by BMJ.

For numbered affiliations see end of article.

Correspondence to Dr Suneth Agampodi; sunethagampodi@yahoo.com

\section{ABSTRACT}

Introduction Sri Lanka has one of the highest incidences of leptospirosis worldwide. We hypothesised that different geographical locations and patient context will have a distinct molecular epidemiology of leptospirosis, based on microgeographical characteristics related to regionaspecific Leptospira predominance. Our objective is to characterise the clinical, epidemiological and molecular aspects of leptospirosis in Sri Lanka to understand disease progression, risk factors and obtain isolates of Leptospira.

Methods and analysis We designed a multicentre prospective study in Sri Lanka to recruit undifferentiated febrile patients and conduct follow-ups during hospital stays. Patients will be recruited from outpatient departments and medical wards. This study will be conducted at two main sites (Anuradhapura and Peradeniya) and several additional sites (Awissawella, Ratnapura and Polonnaruwa). Blood and urine will be collected from patients on the day of admission to the ward or presentation to the outpatient department. Bedside inoculation of 2-4 drops of venous blood will be performed with Ellinghausen-McCullough-Johnson-Harris (EMJH) semisolid media supplemented with antibiotics. Regionally optimised microscopic agglutination test, culture and qPCR-evidence will be performed to confirm the presence of Leptospira in blood which in turn will confirm the presence of disease. Whole genome sequencing will be carried out for all isolates recovered from patients. Multilocus sequence typing (MLST) will be used for the genotyping of new isolates. Sri Lankan isolates will be identified using three published MLST schemes for Leptospira.

Ethics and dissemination Ethical clearance for the study was obtained from Ethics Review Committees (ERC), Medicine and Allied Sciences (FMAS), Rajarata University of Sri Lanka (RUSL) and University of Peradeniya. All genomic data generated through this project will be available at GenBank. Anonymised data will be deposited at the ERC, FMAS, RUSL.

\section{Strengths and limitations of this study}

- This is a multicentre study involving wet and dry zones, low and highlands and high endemic and low endemic areas of the country covering different geographical and climate zones to provide a better understanding of leptospirosis in Sri Lanka.

- We will use a prospective study design focusing on culture isolation as a main aim with specific protocols for the particular objective to describe the strain diversity of Leptospira spp. in Sri Lanka.

- Clinical and epidemiological data will be collected as primary data to ensure high quality data using clinically trained health professionals.

- Since Leptospira are fastidious organisms, the culture yield will be low and linking the clinical disease with isolated Leptospira may be partly confounded by the growth potentials of different Leptospira.

- Since PCR and culture-based methods are typically valid only during the first $7-10$ days of the illness and late presentations may lead to reduced sensitivity of these tests, microscopic agglutination test (MAT) is best interpreted with paired samples, and a lack of convalescent samples will impair the interpretation of MAT results.

\section{INTRODUCTION}

Leptospirosis is a globally widespread, neglected and emerging zoonotic disease ${ }^{1}$ with estimated 1.03 million cases and 58900 annual deaths attributed to the disease. ${ }^{2}$ An average of 2.9 million daily adjusted life years are estimated to be lost every year due to leptospirosis. ${ }^{3}$ Emerging leptospirosis mostly affects vulnerable communities living in resource-poor settings. Often, the disease is either not suspected or is under or misdiagnosed due to the need for laboratory resources to confirm leptospirosis; typically, such resources are neither accessible 
nor affordable. From the clinical perspective, a better understanding of the clinical disease associated with diverse Leptospira species is required and superior diagnostics are needed to prevent severe complications and death. From the public health perspective, the lack of reliable and efficient diagnostic tests makes assessing the burden of disease-whether regionally or globally-difficult.

Sri Lanka has emerged as a country with a high incidence of leptospirosis since $2007^{4}$ and in 2008, the total number of clinically suspected cases reported to the surveillance system was 7406 with 204 deaths. ${ }^{5}$ In 2009, 4980 cases and 145 deaths were reported, ${ }^{6}$ and the outbreak persisted until 2013 with more than 4000 reported cases each year. ${ }^{7}$ The probable case incidence during this time period was more than 20 per 100000 population, indicating that Sri Lanka has one of the highest reported incidences of leptospirosis worldwide. ${ }^{8}$ Our extensive work on the 2008 outbreak suggested that clinical diagnosis of leptospirosis is highly inaccurate, since as much as $40 \%$ of the leptospirosis patients are misdiagnosed in hospitals. ${ }^{9}$ However, all Sri Lankan studies, including our previous study, have the same drawback, as observed in most of the published reports in global literature: hospital admission bias, where a large number of patients with leptospirosis may have been treated as outpatients which leads to a severe underestimate of the actual disease burden. A precise disease burden estimate requires seroprevalence studies coupled with population-based incidence studies.

Our studies on the 2011 outbreak of leptospirosis in Anuradhapura showed that the molecular epidemiology, disease transmission and clinical manifestations of this outbreak were different from those observed in Central Province (wet zone) in 2008. ${ }^{10}$ One of the main concerns regarding these marked variations in the Leptospira strain and the clinical disease was whether this was due to microgeographical variations, environmental conditions or reservoir mammal infection. It has been shown for other diseases such as malaria ${ }^{11} 12$ and schistosomiasis, ${ }^{13-15}$ that microgeography may have a major influence on disease epidemiology. Geochemistry is a well-known and major contributory factor in human health. ${ }^{16}$ Despite the extensive literature available on leptospirosis, studies on the microgeographical variation of Leptospira are scarce.

A systematic review performed on Sri Lankan isolates showed that more than 40 strains from 20 serovars of Leptospira have been reported from Sri Lanka. ${ }^{17}$ Our previous studies on the 2008 and 2011 outbreaks of leptospirosis in Sri Lanka expanded the knowledge of circulating Leptospira and were consistent with a diversity of Leptospira infection in Sri Lanka. We showed that in the 2008 outbreak, L. interrogans was the predominant species, compared with the post-flood outbreak in 2011, in which L. krischneri was the predominant species. However, the molecular epidemiology of Leptospira species in endemic settings remains unknown in
Sri Lanka. In addition to the single tube nested PCR (STNPCR) method, for the first time, we showed that previously published multilocus sequence typing (MLST) schemes could be used with modifications for direct patients samples to study the genetic diversity of infecting Leptospira species in resource-poor settings, where the facilities needed to perform cultures and isolations are minimal. ${ }^{18}$

One of the most important conclusions we draw from our published work is that $\mathrm{PPCR}$ is promising as a clinical diagnostic tool in the diagnosis of leptospirosis during the acute phase, with a wider window of positivity than previously noted. ${ }^{19}$ The editorial by Katz ${ }^{20}$ endorsed the qPCR approach but also emphasised the need for a prospective evaluation of this method. However, most countries with high leptospirosis burdens still find it prohibitively expensive to have molecular based methods for rapid diagnosis, in terms of both the costs and logistics.

Disease burden assessment, public health interventions and clinical management of leptospirosis are challenges due to the lack of diagnostic facilities. Any population-based attempt to estimate the disease burden is often limited by the unavailability of diagnostic tests or the poor performance of the available diagnostic tests. The development of diagnostic tests that are both global and country-specific requires knowledge on circulating serovars. Furthermore, well-characterised samples are required with species and subspecies level identification of infecting Leptospira backed by culture isolations. In Sri Lanka, a culture isolation of Leptospira has not been reported since the 1970s. Even though we showed the microgeographical changes of leptospirosis in 2014, ${ }^{10}$ no prospective studies have been conducted in a Sri Lankan cohort to primarily identify the disease diversity with specific research design. We hypothesised that a different study site and patient context-inpatient vs outpatient-will have a distinct molecular epidemiology of leptospirosis, based on microgeographical characteristics related to region-specific Leptospira predominance, clinical characteristics related to pathogenesis/virulence potential of specific Leptospira species, strains, serovars and the acquisition of infection related to occupational and activities of daily living, reflecting different environmental exposure contexts. Based on these background analyses and hypotheses, the objectives of the present study were as follows:

1. To determine the prevalence of leptospirosis among undifferentiated febrile patients (inpatient and outpatient) in contrasting geographical settings in Sri Lanka.

2. To describe the full clinical spectrum of leptospirosis in patients infected with Leptospira.

3. To determine the predictors of leptospirosis progression using a nested case control approach among febrile patients, both inpatient and ambulatory.

4. To determine the Leptospira species and type diversity in different geographical settings in Sri Lanka.

5. To characterise Sri Lankan Leptospira using MLST and whole genome sequencing methods. 


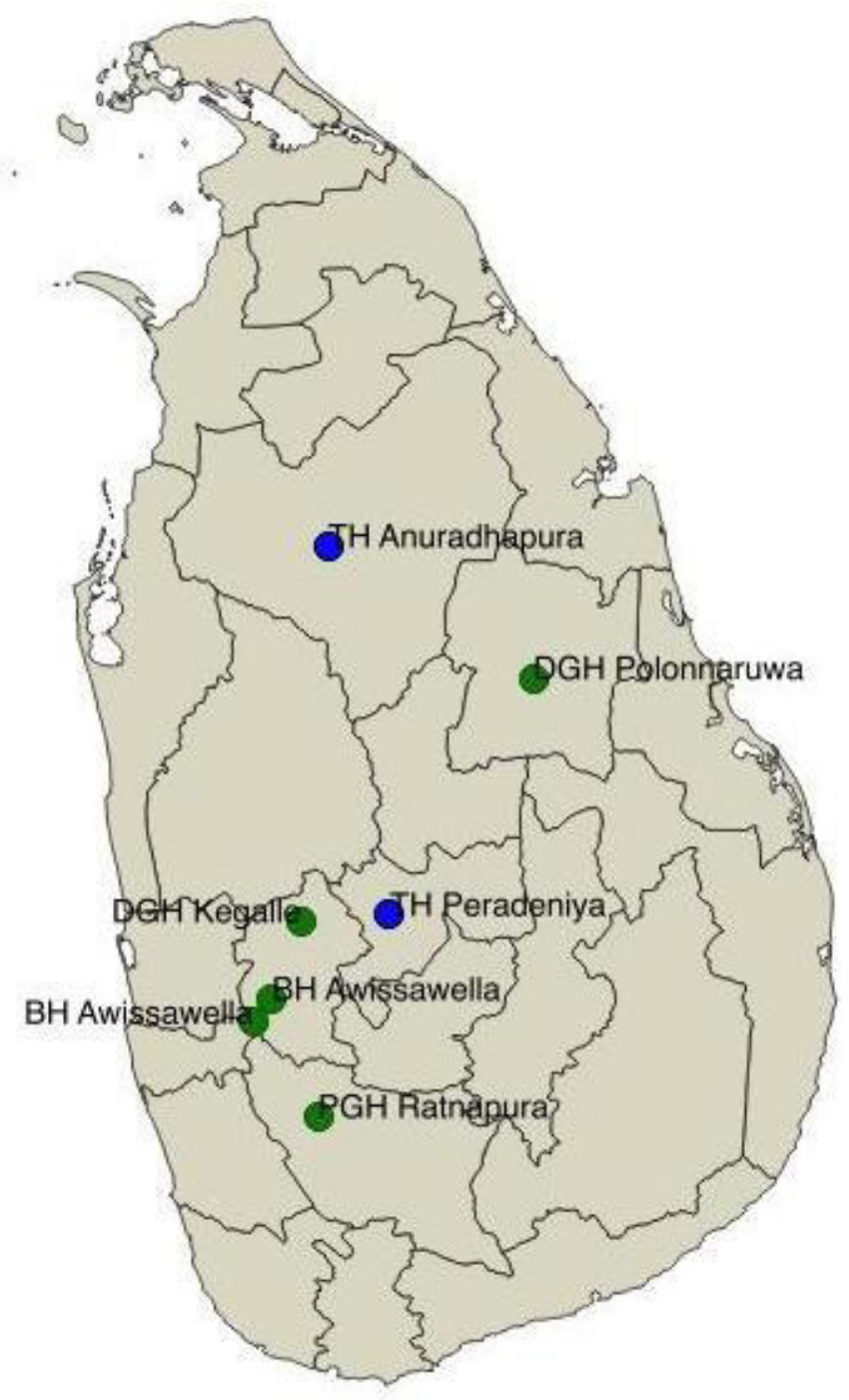

Figure 1 Locations of study sites.

6. To have a well-characterised collection of serum, whole blood and urine samples for the future development and validation of leptospirosis diagnostics.

\section{METHODS AND ANALYSIS}

\section{Study sites}

This study will be performed in four primary subject enrolment/data collection centres in two main contrasting sites: Kandy and Anuradhapura. In addition, a small component of the study will be carried out in Sabaragamuwa, Western, North Central and Southern provinces for species diversity (figure 1). Kandy and Anuradhapura was selected based on our previous observations of Leptospira diversity in these two settings, supported by historically distant identification of diverse Leptospira in Sri Lanka (1960s). ${ }^{21}$ Since no recent studies have been conducted, this project will yield up-to-date information at the molecular and genomically-determined taxonomical levels in addition to conventional methods of identifying Leptospira. The initial focus is on Anuradhapura, located in North Central province of Sri Lanka, in the dry zone of the country. The annual rainfall is $1200-$ $1600 \mathrm{~mm}$, with a mean annual temperature of $\sim 30^{\circ} \mathrm{C}$. In Anuradhapura, paddy farming is carried out by traditional, full-time farmers and constitutes the main mode of income. Cattle, water buffalo, certain rodent specieswell-known sources of Leptospira transmission-are found in and around the paddy fields. Paddy fields are large and well maintained. There are no wetlands or marshy lands in these areas, except the paddy fields during working seasons. Paddy field work depends on irrigation systems, such that, between farming seasons, the paddy fields become completely dry. Other risk factors for acquiring leptospirosis include daily activities involved in living in economically poor conditions, for example, living in proximity to dogs and rodents in houses and neighbourhoods and the wearing of shoes that are often no more than sandals.

The soil structure, water quality/hardness and ecological systems in Anuradhapura contrast with those of the wet zone of Sri Lanka. Kandy (another historic site in Sri Lanka/Ceylon, the cultural capital of the country since ancient times) is situated in the wet zone, with over $3000 \mathrm{~mm}$ of rainfall, and is located in the middle, hilly parts of the country. Paddy farming in this area is based on rainwater and the paddy fields remain wet throughout the year. These are small fields adjacent to wildlife and the soil and ecological structure is different from that in Anuradhapura. Nonetheless, the economic conditions of residents in this region are poor and rural, such that rodent-transmitted and dog-transmitted Leptospira in the context of activities of daily living is likely common but completely unexplored.

The purpose of including additional sites is primarily to identify differences in leptospirosis outbreaks, especially during floods and unsuspected outbreaks. Only selected components of the study will be performed in those areas if there are outbreaks.

\section{Study population}

The study population will include all undifferentiated fever cases either presented to the outpatient department (OPD) or admitted to medical wards/intensive care units (ICUs) in teaching hospita (TH) Anuradhapura and TH Peradeniya (main sites) and selected hospitals in Western, Sabaragamuwa and Southern provinces.

\section{Inclusion criteria}

- Age $\geq 12$ years.

- Temperature $\geq 38^{\circ} \mathrm{C}$.

- Self-reported fever $\geq 2$ days.

\section{Exclusion criteria}

- Fever with running nose without any other symptom or sign.

- Any patient with confirmed diagnosis/foci of infection as a cause for the fever.

- Traumatic or post-operative fever per physician discretion. 
- Fever due to nosocomial infections.

\section{Patient recruitment}

Graduates with a Bachelor of Medicine and Bachelor of Surgery awaiting their internship appointment and established registrars will be assigned to all four centres and directly supervised on a daily basis. They will screen all febrile patients and perform directed physician examinations in the OPD, as well as on inpatients admitted to the medical wards. Once the diagnostic facilities are established, we will inform all clinicians in the selected districts about the available facilities. If they express interest, diagnostic facilities, including cultures to determine species diversity will be provided.

\section{Procedure}

All possible undifferentiated fever cases will be interviewed and examined by the on-site pre-intern medical officers and suspected leptospirosis cases (fulfilling inclusion and exclusion criteria) will be referred to the study project. In the OPD, once the medical officers complete the history taking and examination, the data collector will collect the data. For inward patients, the pre-intern medical officer will screen all patients with fever on admission to the ward, for the selection of eligible patients. In Anuradhapura, four medical units are available and the study will be carried out in all four medical wards. In Peradeniya, a single medical ward is available. Data collectors will visit all wards and ICUs daily for the purpose of data collection. Once the routine ward procedures are completed, data will be collected. All eligible patients will be provided an explanatory statement of the study. The data collector will also explain the benefits and data confidentiality, as well as the right to withdraw from the study at any given time. Once a patient is well informed, written consent will be obtained before enrolment. Data will be collected in the ward, at the patient bed side to ensure the patient's privacy and confidentiality, as a routine clinical procedure. In the OPD, a separate location will be used to collect the data. Data will be collected using a fully structured, interviewer-administered questionnaire. Once the initial data collation is completed, all biological sample will be collected (see the section on sample collection, procession and storage procedure). An additional clinical data check list will be used to assess the clinical features. A daily follow-up will be performed for all hospitalised patients to observe the clinical progress of the disease using a clinical checklist. The questionnaires and the checklists will be adopted from previously published timetested protocols. ${ }^{22}$

All patients will be given an appointment for follow-up 3 weeks after the initial assessment. To maximise patient follow-up, reminders and telephone calls will be used to contact participants before their appointment, and the fee for transportation will be paid. The main purpose of the follow-up would be to obtain a sample for disease confirmation.

\section{Sample size}

Assuming that at least $70 \%$ of patients are infected with same infective Leptospira strain in a specific setting, at least 24 patients with leptospirosis with molecular identification are needed from each site. This calculation was performed with a power of $80 \%$ and an alpha value of 0.05 . Based on our previous studies, the interpretable sequencing data are available for around $50 \%$ of confirmed cases of leptospirosis ${ }^{19}$ and leptospirosis accounts for $15 \%$ of acute undifferentiated fever. ${ }^{23}$ Based on these assumptions, the total number of febrile patients that is required from one sample collection site for this study is 320 . This number of patients is conservatively estimated and feasible to obtain within the project period.

\section{Variables and epidemiological data}

We will be looking at socio-demographic, exposure and clinical data for the clinic-epidemiological components of the study. The sociodemographic data will include age, sex, ethnicity, religion, occupation, income and residence. These variables are usually the distance determinants of leptospirosis and considered as important in determining the social determinants of the disease. Exposure variables will be looking at several types of exposure. First set of variables will include the residential and working environment and hosts. Next set will include actual individual exposure during last 3 weeks. Clinical data will be obtained initially to describe the presenting complaints. All symptoms will be recorded as described by the patients. We will be using a set of probing questions to clarify the presenting complaints. Signs will be documented after the physical examination by the clinically qualified data collector. If a sign is documented after the admission by a treating physician it will also be included, even if it is not demonstrable at the time of examination.

\section{Sample collection, procession and storage procedure}

Blood and urine samples will be collected from patients on the day of admission to the ward or on presentation to the OPD. A total of $7 \mathrm{~mL}$ of blood will be collected and a bed side inoculation of 2-4 drops $(100-500 \mu \mathrm{L})$ will be performed using two tubes containing $9 \mathrm{~mL}$ of EMJH semisolid media with added antibiotics (5-fluorouracil and neomycin). Collected blood added to EMJH media for culture will be incubated at $30^{\circ} \mathrm{C}$ in an incubator.

Venous blood $(2.5 \mathrm{~mL})$ will be collected in an EDTA tube and the remainder will be placed into plain tubes for serum separation. For serum separation, the blood in the plain tubes will be centrifuged at $1300 \mathrm{rpm}$ for $10 \mathrm{~min}$. The blood in the EDTA tubes will be used for PCR assay and the serum samples for serological assays. Aliquots $(500 \mu \mathrm{L})$ will be prepared from the whole blood and serum samples. These will then be frozen at $-20^{\circ} \mathrm{C}$ or $-80^{\circ} \mathrm{C}$ for short-term or long-term storage, respectively. This procedure will be completed within 2 hours.

Clean catch mid-stream $(10-15 \mathrm{~mL})$ urine will be collected into a sterile wide mouth container and processed within 2 hours of collection. Initially, the urine 
Table 1 Criteria for the diagnosis of confirmed and probable cases of leptospirosis

\begin{tabular}{ll}
\hline Case & Definition \\
\hline Confirmed & Clinical signs and symptoms consistent with leptospirosis with any one of the following criteria: \\
& 1. Fourfold increase in MAT titre in acute and convalescent serum samples. \\
& 2. MAT titre $\geq 1: 400$ in single or paired serum samples. \\
& 3. Isolation of pathogenic Leptospira species from normally sterile sites. \\
4. Pathogenic Leptospira species DNA detected by PCR. & Clinical signs and symptoms consistent with leptospirosis with any one of the following criteria: \\
& 1. Presence of IgM antibodies by ELISA or dipstick. \\
& 2. MAT titre $\geq 1: 100$ in single acute-phase serum sample (with no convalescent serum).
\end{tabular}

MAT, microscopic agglutination test.

will be centrifuged at $3000 \mathrm{rpm}$ for $5 \mathrm{~min}$ to sediment the white cells, epithelial cells and other crystals. Next, the supernatant will be separated and further centrifuged at $15000 \mathrm{rpm}$ for $10 \mathrm{~min}$. The sediment obtained will be used for PCR studies and stored at $-20^{\circ} \mathrm{C}$ until further use. $^{24-26}$ The available protocols for qPCR based urine analysis will be tested and optimised. If the urine cannot be processed during the specified time period, the $\mathrm{pH}$ of the urine will be adjusted to approximately 7.4 using 1 $\mathrm{M} \mathrm{NaOH}$ solution. Three weeks after the onset of fever, a follow-up blood sample $(2 \mathrm{~mL})$ will be collected in plain tubes for paired sera and $10-15 \mathrm{~mL}$ of urine will be collected for PCR assay.

\section{Disease confirmation}

Disease will be confirmed using a regionally optimised microscopic agglutination test (MAT), culture and qPCR-evidence of the presence of Leptospira in the serum. The criteria for confirmed and probable cases of leptospirosis is presented in table $1 .^{27}$

\section{Microscopic agglutination test}

For this purpose, we will use the US Center for Disease Control's MAT panel with five already available local strains obtained from the Royal Tropical Institute, Amsterdam (KIT). Once the Sri Lankan isolates are obtained, a validation of best panel suitable for Sri Lanka will be determined. MAT will be carried out in two main steps. First, the serum samples will be screened at a titre of 1/50 using the MAT panel of 24 strains, which includes the five local strains. Positive sera from the screening test will be subjected to a run out test with the serial dilution of sera from $1 / 50$ to $1 / 3200$. MAT evidence for seropositivity will be defined as one or more of the following: seroconversion from negative to positive $(<1 / 50$ to $>1 / 50)$; fourfold increase in titre between acute-phase and convalescent-phase (follow-up) samples; or a single titre of $\geq 400$.

\section{Culture isolation of Leptospira}

For the culture isolation, we adopted the previously validated protocol published by Wuthiekanan et al. ${ }^{28} \mathrm{EMJH}$ semisolid media will be prepared by adding $2.3 \mathrm{~g}$ of EMJH base, $1.5 \mathrm{~g}$ of bacteriological agar and $100 \mathrm{mg}$ of sodium pyruvate into $785 \mathrm{~mL}$ of distilled water, then adjusting the $\mathrm{pH}$ to 7.4. The media will be autoclaved and cooled to around $50^{\circ} \mathrm{C}$, Next, $100 \mathrm{~mL}$ of Leptospira enrichment media and $100 \mathrm{~mL}$ of fetal bovine serum will be added to the media. ${ }^{29-32}$ To suppress the growth of possible contaminants, 5-fluorouracil and neomycin will be added, at concentrations of $100 \mu \mathrm{g} / \mathrm{mL}$ and $25 \mu \mathrm{g} / \mathrm{mL}$, respectively. The media inoculated with blood will be inspected using dark field microscopy for the presence of motile Leptospira, initially after 3 weeks and then on a monthly basis. When a positive growth is detected, sub cultures will be transferred into liquid and semisolid media. An aliquot will then be obtained and fixed with 5\% DMSO (dimethyl sulfoxide) and stored in $-80^{\circ} \mathrm{C}$. To maintain live cultures, the isolates will be subcultured in liquid media on a bi-weekly basis and on semisolid media every 3 months. Serotyping of the newly isolated Leptospira strains will be carried out at the Pasteur Institute, France.

\section{Quantitative PCR}

\section{Diagnosis}

For the diagnosis, DNA will be extracted from whole blood, serum and urine samples using the QIAamp DNA Blood Mini Kit (Qiagen, USA), according to the manufacturer's instructions. The extracted DNA will be quantified using an Invitrogen Qubit 4 Flurometer. Two previously published quantitative PCR protocols targeting 16 s ribosomal RNA and Lipl32 genes will be used for the detection of pathogenic Leptospira DNA based on SYBR Green Chemistry (table 2). ${ }^{33}$

\section{Speciation}

To determine the Leptospira species in the positive cultures, a previously published quantitative PCR protocol based on SYBR Green Chemistry (table 3) will be used (before sequencing). ${ }^{34}$ This will facilitate the identification of $\mathrm{L}$. interrogans, L. borgpetersenii, L. kirschneri and L. noguchii.

\section{Molecular studies}

For newly isolated Leptospira, whole genome sequencing will be performed using the following two technologies: MinION Nanopore sequencing in the Leptospirosis Research Laboratory (Faculty of Medicine and Allied Sciences, Rajarata University of Sri Lanka) and PacBio third generation sequencing at the Institute for Genomic 
Table 2 Primers for the detection of Leptospira DNA in clinical samples

\begin{tabular}{lll} 
Primer/probe sequence & Annealing temperature & Complementary target species \\
\hline Forward: 5'-AAG CAT TAC CGC TTG TGG TG-3' & $60^{\circ} \mathrm{C}$ & lipL32 gene of pathogenic Leptospira spp. \\
Reverse: 5'-GAA CTC CCA TT CAG CGA TT-3' & & \\
Forward: 5'-TAA AGG CTC ACC AAG GCG AC-3' & $60^{\circ} \mathrm{C}$ & 16 s gene of pathogenic Leptospira spp. \\
Reverse: 5'-TTA GCC GGT GCT TTA GGC AG-3' & &
\end{tabular}

Medicine (Universiy of California, San Diego, USA) or in a place where it is available.

BAS and BAX files containing all the DNA sequence information will be converted into FASTA files. Prior to assembly, the quality (coverage) of the raw data will be assessed. Using the PacBioEDA software, the quality summary of the data will be obtained. The CanuPacBiO assembly programme will be used for genome assembly. The raw data of BAS/BAX files will be converted into bam files using the bax2bam software. These bam files will be aligned to our references using the Pbalingn software. The software Quiver will be used to polish the data. The assembled data will be annotated using Prokka ${ }^{35}$ and Roary, ${ }^{36}$ and annotations will be inspected and curated using Artemis. The functional annotation will be performed using the eggNOG and eggNOG MAPPER online tools. ${ }^{37}$ The genomes of the Sri Lankan isolates will be compared using MAUVE software. The organism will be roughly identified from the direct raw data using KRAKEN. ${ }^{38}$

MLST will be used for the genotyping of the new isolates. Sri Lankan isolates will be identified using three published MLST schemes for Leptospira. ${ }^{39-41}$ Genetic similarity between the isolates will be determined using SPLITSTREE.

\section{Taxonogenomics, pan-genome and phylogenetic analyses}

To establish how this strain was related to other Leptospira strains, we will use several genomic analysis tools. First, the 16S rRNA sequences will be used to construct a phylogeny. High sequence identities $(\geq 97 \%)$ between the newly sequenced strain and previously identified Leptospira strains will be preliminarily used as suggestive of phylogenetic relationships. Accordingly, the $16 \mathrm{~S}$ rRNA gene sequences of Leptospira spp. deposited in the GenBank database will be acquired. Multiple sequence alignment based on 16S rRNA will be performed using ClustalX
V.1.81. These results will be then used to construct the maximum likelihood tree using MEGA V.5.05. To evaluate clade support, we will perform a bootstrap analysis with 1000 replicates. The relationship of each sequenced genome to the previously described or novel Leptospira species will be determined according to the Overall Genetic Relatedness Indices, Average Nucleotide Identity (ANI) and the Average Amino Acid Identity. The indices will be automatically calculated using two-way BLA blastn and blastp. For the ANI calculation, the default parameters shall be as follows: sequence identity cut-off, $30 \%$; alignment cut-off, 70\%; query length, $1020 \mathrm{bp}$.

\section{Data management and analysis}

Patient interviews and patient health records will be used as data sources. On-site data entry will be performed in real-time for all the collected data. An EpiInfo database will be prepared using cross-checks, field validation and other in-built techniques available for data quality improvement. The investigators will frequently visit the hospitals to cross-validate the data collected. All databases will be stored on password-protected computers. The main database will be stored at the Leptospirosis Research Laboratory of the Faculty of Medicine and Allied Science, Rajarata University of Sri Lanka. Personal identification data will be removed from all the databases, such that only aliquots and predesigned patient identifiers are available. Hardcopies of the questionnaires will be archived using the standard protocols for archiving hard copies of questionnaires. Only the investigators will have access to the database. The database will be continuously updated with laboratory data using a linking patient identifier (key variable). The laboratory results will be generated for all samples daily, once a fully functioning laboratory is set up, and the treating physicians will be informed about this over the phone. Separate laboratory registers will be

Table 3 Primer pairs for detection of species

\begin{tabular}{lll}
\hline Primer/Probe sequence & Annealing temperature & Complementary target species \\
\hline $\begin{array}{l}\text { Forward: 5'-CTT GAG CCT GCG CGT TAY-3' } \\
\text { Reverse: 5'-CCG ATA ATT CCA GCG AAG ATC-3' }\end{array}$ & $63^{\circ} \mathrm{C}$ & secY gene of $L$. interrogans \\
Forward: 5'-GAT TCG GGT TAC AAT TAG ACC-3' & $65^{\circ} \mathrm{C}$ & ompL1 gene of L. borgpetersenii \\
Reverse: 5'-TTG ATC TAA CCG GAC CAT AGT-3' & & secY gene of \\
Forward: 5'-CTG GCT TAA TCA ATG CTT CTG-3' & $60^{\circ} \mathrm{C}$ & L. kirschneri \\
Reverse: 5'-CTC TT CGG TGA TCT GTT CC-3' & & secY gene of L. noguchii \\
Forward: 5'-TCA GGG TGT AAG AAA GGT TC-3' & $63^{\circ} \mathrm{C}$ & \\
\hline Forward: 5'-CAA AAT TAA AGA AGC AAA GA-3' & & \\
\hline
\end{tabular}


maintained for the receipt of samples and the delivery of the results.

For the missing data, case deletions and pairwise deletions will be carried out, based on the amount of missing data. Proportions and 5\% confidence intervals will be used to describe the confirmed cases, diversity of infecting Leptospira and sequelae. A $\chi^{2}$ test will be used to identify any trends and test significant differences in the Leptospira diversity in the different microgeographical and clinical settings. Predictors of severe disease will be analysed using a logistic regression model. Model building will be performed using a conceptual hierarchy of determinants, which we have previously proposed for leptospirosis. An online database for Leptospira MLST will be used for typing analysis.

\section{Patient and public involvement}

The study was developed based on the patients' feedback regarding leptospirosis disease burden, its impact on their lives and in particular, the problems faced during illness. In previous studies, we specifically explored patients as well as general public views on leptospirosis disease and prevention, and used this information for the design of this study. Moreover, the results of this study will be disseminated on a continuous basis for the patients. We have already conducted several public awareness and health worker education workshops based on our findings.

\section{ETHICS AND DISSEMINATION}

Undifferentiated fever patients eligible for the study will be given an explanatory statement of the study and written informed consent will be obtained before enrolment. Rapid diagnostic kits will be used on site to assist treating physicians. qPCR diagnostic facilities will be provided for all patients whenever the urgent tests are requested by treating physicians. All test results will be informed to the patients and the wards.

All genomic data will be deposited in Gene Bank. Characterised serum samples will be stored in $-80^{\circ} \mathrm{C}$ in the Faculty of Medicine and Allied Sciences, Rajarata University of Sri Lanka. Data pertaining to this study will be available for researchers and policy-makers on a reasonable request address to the corresponding author. After the completion of work, data will be deposited in a repository.

\section{Author affiliations}

${ }^{1}$ Department of Community Medicine, Faculty of Medicine and Allied Sciences, Rajarata University of Sri Lanka, Saliyapura, Sri Lanka

${ }^{2}$ Department of Microbiology, Faculty of Medicine and Allied Sciences, Rajarata University of Sri Lanka, Saliyapura, Sri Lanka

${ }^{3}$ Department of Biochemistry, Faculty of Medicine and Allied Sciences, Rajarata University of Sri Lanka, Saliyapura, Sri Lanka

${ }^{4}$ Department of Microbiology, Faculty of Medicine, University of Peradeniya, Peradeniya, Sri Lanka

${ }^{5}$ Department of Medicine, Faculty of Medicine, University of Peradeniya, Peradeniya, Sri Lanka

${ }^{6}$ Department of Medicine, Faculty of Medicine and Allied Sciences, Rajarata University of Sri Lanka, Saliyapura, Sri Lanka
${ }^{7}$ Yale University School of Medicine, New Haven, Connecticut, USA

Contributors JV and SA drafted the initial protocol. JW, DJ and IS are responsible for the detailed design, field/ laboratory work analysis and interpretation of objectives 1-3, 4 and 5, respectively. CG, SK and SS helped in design, field, hospital and laboratory work. MM participated in scientific design and guided all laboratory components. All authors have read and approved the manuscript.

Funding This project is the Sri Lankan component of a multicountry study funded by the NIAID of the National Institutes of Health under award number U19Al115658. Funders had no role in the design, execution, analyses, data interpretation or decision to submit results.

Map disclaimer The depiction of boundaries on the map(s) in this article do not imply the expression of any opinion whatsoever on the part of BMJ (or any member of its group) concerning the legal status of any country, territory, jurisdiction or area or of its authorities. The map(s) are provided without any warranty of any kind, either express or implied.

Competing interests None declared.

Patient consent for publication Not required.

Ethics approval Ethical clearance for the study was obtained from the Ethic Review Committees of Faculty of Medicine and Allied Sciences, Rajarata University of Sri Lanka (ERC/2015/18).

Provenance and peer review Not commissioned; externally peer reviewed.

Open access This is an open access article distributed in accordance with the Creative Commons Attribution Non Commercial (CC BY-NC 4.0) license, which permits others to distribute, remix, adapt, build upon this work non-commercially, and license their derivative works on different terms, provided the original work is properly cited, appropriate credit is given, any changes made indicated, and the use is non-commercial. See: http://creativecommons.org/licenses/by-nc/4.0/.

\section{REFERENCES}

1. Leptospirosis worldwide, 1999. Wkly Epidemiol Rec 1999;74:237-42.

2. Costa F, Hagan JE, Calcagno J, et al. Global morbidity and mortality of leptospirosis: a systematic review. PLoS Negl Trop Dis 2015;9:e0003898.

3. Taylor AJ, Paris DH, Newton PN. A systematic review of the mortality from untreated leptospirosis. PLoS Negl Trop Dis 2015;9:e0003866.

4. Agampodi S, Peacock SJ, Thevanesam V. The potential emergence of leptospirosis in Sri Lanka. Lancet Infect Dis 2009;9:524-6.

5. Epidemiological unit: selected notifiable diseases reported by medical officers of health. Weekly Epidemiological Report 2009;36.

6. Epidemiological unit: selected notifiable diseases reported by medical officers of health. Weekly Epidemiological Report 2010;37.

7. Warnasekara JN, Agampodi S. Leptospirosis in Sri Lanka. Sri Lankan Journal of Infectious Diseases 2017;7.

8. Pappas G, Papadimitriou P, Siozopoulou V, et al. The globalization of leptospirosis: worldwide incidence trends. International Journal of Infectious Diseases 2008;12:351-7.

9. Agampodi SB, Peacock SJ, Thevanesam V, Burns MA, et al. Leptospirosis outbreak in Sri Lanka in 2008: lessons for assessing the global burden of disease. Am J Trop Med Hyg 2011;85:471-8.

10. Agampodi SB, Dahanayaka NJ, Bandaranayaka AK, et al. Regional differences of leptospirosis in Sri Lanka: observations from a floodassociated outbreak in 2011. PLoS Negl Trop Dis 2014;8:e2626.

11. Myers WP, Myers AP, Cox-Singh J, et al. Micro-geographic risk factors for malarial infection. Malar J 2009;8:27.

12. Schellenberg J, Newell JN, Snow RW. An analysis of the geographical distribution of severe malaria in children in Kilifi district, Kenya. Int J Epidemiol 1998;27:323-9.

13. Hassan AO, Amoo AOJ, Deji-Agboola AM, et al. Hassan Ro: Microgeographical patterns of schistosomiasis and anthropometric indices of children in eko-ende South West, Nigeria. Int J Biol Med Res 2911;2:656-60.

14. Kloos H, Gazzinelli A, Van Zuyle P. Microgeographical patterns of schistosomiasis and water contact behavior; examples from Africa and Brazil. Mem. Inst. Oswaldo Cruz 1998;93(suppl 1):37-50.

15. Baliraine FN, Afrane YA, Amenya DA, et al. High prevalence of asymptomatic Plasmodium falciparum infections in a highland area of Western Kenya: a cohort study. J Infect Dis 2009;200:66-74.

16. Dissanayake CB, Chandrajith R. Medical geochemistry of tropical environments. Earth Sci Rev 1999;47:219-58.

17. Naotunna C, Agampodi SB, Agampodi TC. Etiological agents causing leptospirosis in Sri Lanka: a review. Asian Pac J Trop Med 2016;9:390-4. 
18. Agampodi SB, Moreno AC, Vinetz JM, et al. Utility and limitations of direct multi-locus sequence typing on qPCR-positive blood to determine infecting Leptospira strain. Am J Trop Med Hyg 2013;88:184-5.

19. Agampodi SB, Matthias MA, Moreno AC, et al. Utility of quantitative polymerase chain reaction in leptospirosis diagnosis: association of level of leptospiremia and clinical manifestations in Sri Lanka. Clin Infect Dis 2012;54:1249-55.

20. Katz AR. Quantitative polymerase chain reaction: filling the gap for early leptospirosis diagnosis. Clin Infect Dis 2012;54:1256-8.

21. Nityananda K. Harvey T: leptospirosis in Ceylon-Epidemiological and laboratory studies. Ceylon J Med Sci 1971;20:5-14.

22. Agampodi SB, Nugegoda DB, Thevanesam V. Determinants of leptospirosis in Sri Lanka: study protocol. BMC Infect Dis 2010;10:332.

23. Reller ME, Bodinayake C, Nagahawatte A, et al. Leptospirosis as frequent cause of acute febrile illness in southern Sri Lanka. Emerg Infect Dis 2011;17:1678-84.

24. Lucchesi PMA, Arroyo GH, Etcheverría Al, et al. Recommendations for the detection of Leptospira in urine by PCR. Rev Soc Bras Med Trop 2004;37:131-4.

25. Bal AE, Gravekamp C, Hartskeerl RA, et al. Detection of leptospires in urine by PCR for early diagnosis of leptospirosis. J Clin Microbiol 1994;32:1894-8.

26. Van Eys GJ, Gravekamp C, Gerritsen MJ, et al. Detection of leptospires in urine by polymerase chain reaction. J Clin Microbiol 1989;27:2258-62.

27. World Health Organization. Report of the second meeting of leptospirosis burden epidemiology reference group. Geneva: World Health Organization, 2011: 1-34.

28. Wuthiekanun V, Chierakul W, Limmathurotsakul D, et al. Optimization of culture of Leptospira from humans with leptospirosis. J Clin Microbiol 2007;45:1363-5.

29. Wuthiekanun V, Amornchai P, Langla S, et al. Maintenance of Leptospira species in Leptospira Vanaporn Wuthiekanun agar. J Clin Microbiol 2014;52:4350-2.
30. Wuthiekanun V, Amornchai P, Langla S, et al. Antimicrobial disk susceptibility testing of Leptospira spp. using Leptospira Vanaporn Wuthiekanun (LVW) agar. Am J Trop Med Hyg 2015;93:241-3.

31. Wuthiekanun V, White NJ, Amornchai P, et al. Quality controls for antimicrobial disk diffusion testing on Leptospira Vanaporn Wuthiekanun agar. Trans R Soc Trop Med Hyg 2016;110:673-5.

32. Picardeau M, Bulach DM, Bouchier C, et al. Genome sequence of the saprophyte Leptospira biflexa provides insights into the evolution of Leptospira and the pathogenesis of leptospirosis. PLoS One 2008;3:e1607.

33. Backstedt BT, Buyuktanir O, Lindow J, et al. Efficient detection of pathogenic leptospires using $16 \mathrm{~S}$ ribosomal RNA. PLoS One 2015;10:e0128913.

34. Ferreira AS, Costa P, Rocha T, et al. Direct detection and differentiation of pathogenic Leptospira species using a multi-gene targeted real time PCR approach. PLoS One 2014;9:e112312.

35. Seemann T. Prokka: rapid prokaryotic genome annotation. Bioinformatics 2014;30:2068-9.

36. Page AJ, Cummins CA, Hunt M, et al. Roary: rapid large-scale prokaryote pan genome analysis. Bioinformatics 2015;31:3691-3.

37. Huerta-Cepas J, Forslund K, Coelho LP, et al. Fast genome-wide functional annotation through orthology assignment by eggNOGMapper. Mol Biol Evol 2017;34:2115-22.

38. Wood DE, Salzberg SL. Kraken: ultrafast metagenomic sequence classification using exact alignments. Genome Biol 2014;15:R46.

39. Varni V, Ruybal P, Lauthier JJ, et al. Reassessment of MLST schemes for Leptospira spp. typing worldwide. Infection, Genetics and Evolution 2014;22:216-22.

40. Ahmed N, Devi SM, Valverde MdelosA, et al. Multilocus sequence typing method for identification and genotypic classification of pathogenic Leptospira species. Ann Clin Microbiol Antimicrob 2006;5:28.

41. Boonsilp S, Thaipadungpanit J, Amornchai P, et al. A single multilocus sequence typing (MLST) scheme for seven pathogenic Leptospira species. PLoS Negl Trop Dis 2013;7:e1954. 